\title{
ELECTRICAL PROPERTIES PREDICT WHEAT LEAF MOISTURE
}

\author{
Y. M. Hao, Y. T. Hua, X. Li, X. Q. Gao, J. L. Chen
}

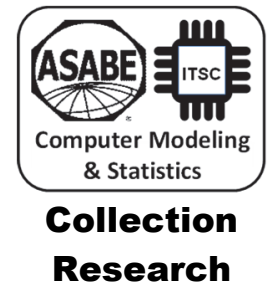

\section{HighLIGHTS}

- A non-destructive prediction model for moisture content of wheat leaves was established based on electrical properties.

- The model based on a single property (capacitance or resistance) was improved by using both properties.

- The model accurately detected the moisture content of wheat leaves in real-time to avoid irrigation lag.

- The results provide a basis for real-time and targeted water-saving irrigation of winter wheat in an arid region.

ABSTRACT. In this study, we aimed to establish a non-destructive and rapid approach to monitor the moisture content of wheat leaves in Southern Xinjiang, China, and promptly acquire information on the physiological water demand of crops to guide precision irrigation. Wheat leaves were used as the research object. Using a custom-made clamped parallel-plate capacitor and LCR digital bridge meter, we determined the electrical properties (capacitance and resistance) of wheat leaves with various moisture contents within a frequency range from 0.12 to $100 \mathrm{kHz}$. Moreover, we explored the correlation between leaf moisture content and the electrical properties. Our data showed that leaf moisture exhibited the best correlation with the electrical properties at $50 \mathrm{kHz}$. Under these optimized conditions, a model for moisture measurement was established using a single-parameter method (capacitance or resistance). However, the estimated standard errors (RMSE) of this method were $3.29 \%$ (for resistance) and $3.49 \%$ (for capacitance), which were greater than the standard error of the measured moisture content (2\%). Therefore, we developed an improved model using a two-parameter method (capacitance and resistance), and the estimated standard error was $2.68 \%$, which was more feasible for predicting moisture content compared with the single-parameter method. The model was validated using eight groups of wheat leaf samples at the turning-green stage and the jointing stage, and the RMSE values were less than 2\%. Our findings provide a scientific basis for real-time and targeted water-saving irrigation of wheat in arid areas of Southern Xinjiang.

Keywords. Electrical property, Model, Moisture content, Precision irrigation, Wheat leaves.

W heat is the main grain crop in Southern Xinjiang, China, and it plays a key role in the agricultural industry. Wheat production is of great significance to increase farm incomes and promote regional development $(\mathrm{Li}, 2014$; Khamparia et al., 2020; Sankar et al., 2020). Southern Xinjiang, located in the south of Xinjiang Province, has a typical temperate continental climate, with annual precipitation of less than 150 $\mathrm{mm}$, serious soil desertification, water resource shortages, and a large irrigated area (Yang and Chen, 2016). Water plays a crucial role in the growth and development of crops. Without enough water, plant performance is impaired (Abayomi and Wright, 1999; Liu, 1994). Agricultural drought limits the development of wheat, leading to a decrease in

Submitted for review on 15 July 2020 as manuscript number ITSC 14210; approved for publication as a Research Article and as part of the Computer Modeling and Statistics for Agriculture Collection by the Information Technology, Sensors, \& Control Systems Community of ASABE on 24 November 2020.

The authors are Yumei Hao, Lecturer, Yuantao Hua, Lecturer, Xu Li, Associate Professor, Xianqiang Gao, Associate Professor, and Jilong Chen, Associate Professor, College of Information Engineering, Tarim University, Alar, Xinjiang, China. Corresponding author: Yuantao Hua, Tarim University, Alar, Xinjiang 843300, China; phone: +86-18895086309; e-mail: hythym520@163.com. yield (Mamnouei et al., 2006; Wang, 2017). Scientific irrigation management can reduce the waste of water resources and is beneficial for the presence and persistence of wheat (Dong et al., 2011; Wang et al., 2016). Therefore, we aimed to develop a new method for predicting crop moisture status as an irrigation index.

The measurement of plant moisture content has a long history. Initial crop moisture loss can be detected indirectly using environmental factors, such as soil moisture (Eller and Denoth, 1996), leaf transpiration rate, rainfall, air temperature, and humidity. Among these factors, detection of soil moisture based on relative dielectric properties has been rapidly developed and is used to guide irrigation by measuring the soil-water dielectric constant to determine the soil moisture status (Dean et al., 1987; Thompson et al., 2007). However, this measurement method is not accurate due to variations in soil properties, the plant root depth, the definition of drought, and calibration of the sensors. All these factors are difficult to define, leading to measurement error and response lag, and finally resulting in inaccurate irrigation (Zhang et al., 1999).

Turner (1990) discussed the development of plant water deficits, stating that plant water deficits are caused by the hydraulic gradients developed in the soil-plant-atmosphere 
continuum, and found that the photosynthetic and transpiration rates of cowpea, sunflower, soybean, and other crops are decreased when two-thirds of the extractable soil water in the root zone has been used. Furthermore, leaf enlargement is more sensitive than leaf photosynthesis to a decrease in extractable soil water, indicating that the soil water status may not be a reliable indicator of plant activity.

Although plant water flows up from the roots, and the roots absorb water from the soil, it is usually difficult to know where to measure the water content and how to integrate the measurement range due to the variation in soil water content within the root zone. Therefore, it is generally believed that the water status or physiological activity of plants is a more reliable indicator for irrigation scheduling than the soil water status. Leaves are also the main waterloss organs of plants, and leaf water potential is considered a good indicator of crop water status. By measuring the soil water content and leaf water potential after each irrigation treatment, it has been found that the leaf water potential does not completely and synchronously change with soil moisture. To a certain extent, it is also affected by changes in environmental conditions (e.g., weather and cultivation conditions) (Gao et al., 2005; Luan et al., 2007).

Drought stress can affect the growth and development of plants. Some studies have found that the photosynthetic and transpiration rates of maize leaves decreased under water stress, and the transpiration rate was more sensitive to water stress than the photosynthetic rate (Liu et al., 2013). Moreover, plants have drought resistance and can adapt to the slow development of water deficit through an osmotic adjustment mechanism. Plants can automatically adjust the opening of leaf stomata (partially closed or completely closed), control the speed of water vapor diffusion, and modify the amount of transpiration loss under drought stress.

Previous researchers (Jin et al., 1999) irrigated corn with different amounts of water to achieve the status of sufficient water, adequate water, or water deficit and then tested the transpiration rate and corresponding physiological resistance of corn leaves in those different irrigation states. The results showed that leaf physiological resistance reflected the water status of the plants more quickly than the soil moisture content. Other studies detected drought stress using the electrical properties of plant leaves with a custom-made parallelplate capacitor that was clamped onto the leaves. The results showed that the water status of leaves could be indicated by the change in capacitance, which may reflect the water deficit of the plant with more sensitivity than other methods (Liu, 2014; Zhang, 2016). From the perspective of plant physiology, the leaf is an important organ for transpiration, nutrient transformation, respiration, and other physiological processes. Therefore, its moisture status can more accurately reflect the crop water demand (Wang and Clarke, 1993; Lu et al., 2005).

Non-destructive inspection is a widely used approach to crop monitoring. Measurement of electrical properties is an important non-destructive method and has been examined extensively due to its simple and rapid operation for moisture detection in plants (Kandala et al., 1989; Afzal et al., 2010; Wang et al., 2013; Llave et al., 2014). For example, Rascio et al. (2019) developed a low-cost humidity sensor to compare electrical resistance estimates of leaf moisture content and a phenotypic protocol for genotypic screening of wheat. Mizukaw et al. (2006) developed a method for measuring the moisture content of tea leaves, showing that capacitance and resistance had a strong influence on the moisture content. Zheng et al. (2015) developed a detector to measure plant electrical properties based on a four-electrode method and tested corn leaves at different growth stages and different levels of water status, demonstrating that the electrical properties of corn leaves can be used to effectively evaluate water stress.

When plants have too little water, the moisture status of their leaves will be reduced due to drought stress. In contrast, when plant have enough water, the moisture status of their leaves will be significantly increased. Therefore, the moisture status of the leaves can be used to detect the moisture status of the plant. Plant leaves are mainly composed of water, cellulose, and other substances. At room temperature, the relative dielectric constant of cellulose is about 3.9, while that of water is about 80 . Therefore, the relative dielectric constant of leaves mainly depends on the moisture status of the leaves. Furthermore, leaves with different levels of moisture status exhibit distinct dielectric constants (Feng et al., 2010; Lu, 2017). When using an electrical property to detect the moisture content of leaves, the leaf samples are used as the dielectric, and the measurement of moisture content is achieved according to the difference between the dielectric constants of leaves with different moisture status. Detection of moisture status based on the electrical properties of plant leaves is an active research field (Wei et al., 2008; Zheng et al., 2015). However, this detection approach typically considers the effect of only a single electrical property (capacitance or resistance) on plant moisture content and rarely considers a two-parameter method (capacitance and resistance). Moreover, few studies have investigated frequency characteristics when measuring moisture using electrical properties.

Based on the above-mentioned reasons, we aimed to study the correlation between the moisture status of wheat leaves and their electrical properties, including the frequency characteristics, and develop a prediction model for moisture status. We also aimed to establish a non-destructive and effective detection method to determine wheat leaf moisture status and understand the indicators of drought stress in wheat crops.

\section{Materials ANd Methods \\ EXPERIMENTAL MATERIAL}

Wheat leaves at the turning-green stage, start-up stage, and jointing stage were selected from crops that were planted on 7 October 2018 at the Agronomy Experimental Station of Tarim University. The selected leaves were used as experimental material during the spring of 2019.

\section{EXPERIMENTAL SETUP}

Sample leaves were collected three times from the end of February to the beginning of April 2019. One week before each sample collection, the test fields were irrigated with 
different amounts of water. Therefore, the initial moisture status of the wheat plants was different, and the test fields were not irrigated in the subsequent experimental process. Due to the water use and evapotranspiration of plants, the water content gradually decreases (Guo et al., 2000). For the turning-green stage of wheat, nine leaves were collected on 28 February 2019. For the start-up stage, nine wheat leaves were collected on 18 March 2019. For the jointing stage, ten leaves were collected on 8 April 2019. To obtain wheat leaves with different moisture status and reduce the measurement error, each sample collection started at 9:00 a.m., the upper unfolded part of the wheat leaves was collected, leaves with large width and similar size were collected, and the surfaces of the leaves were cleaned to reduce the amount of wax. After sample collection, the leaf samples were placed in numbered plastic bags and immediately transported to the laboratory (Tanentzap et al., 2015). Each sample was measured ten times with the custom-made detector, and the mean value was taken as the final result.

\section{Measurement of Electrical Properties}

The equipment used for the experiment included an LCR digital bridge meter (model Victory VC4091C), a short-tail film pressure sensor (model FSR402, Shanghai Qixin Scientific Instrument Co.), an electric constant-temperature blast drying oven (model DHG-9145A, Shanghai Qixin Scientific Instrument Co.), an analytical balance (model FA224TC, Lichen Technology Electronics, indexing value of $0.0001 \mathrm{~g}$ ), and a custom-made clamping parallel-plate capacitor.

The clamping parallel-plate capacitor was designed for measurement of the electrical properties of wheat leaves. Figure 1 shows the structure of the parallel-plate capacitor. The frame of the device was made of acrylic plate. The two circular copper plates each had a diameter of $15 \mathrm{~mm}$ and thickness of $2 \mathrm{~mm}$. The upper plate was connected to the threaded shaft with a plastic insulator, and the lower plate was pressed against the pressure sensor by a plastic insulator, which was fixed on the frame of the device. During measurement, a wheat leaf was gently placed between the two plates, and then the pressure of the plate on the leaf was adjusted by slowly turning the threaded shaft to move the upper

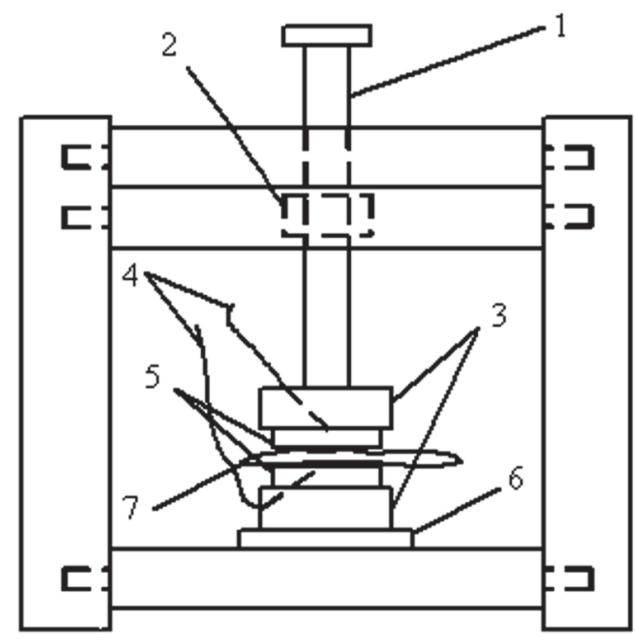

Figure 1. Structure of parallel-plate capacitor: $1=$ threaded shaft, $2=$ nut, $3=$ plastic insulators, $4=$ single-core shielded wires, $5=$ copper electrode plates, $6=$ pressure sensor, and $7=$ wheat leaf.

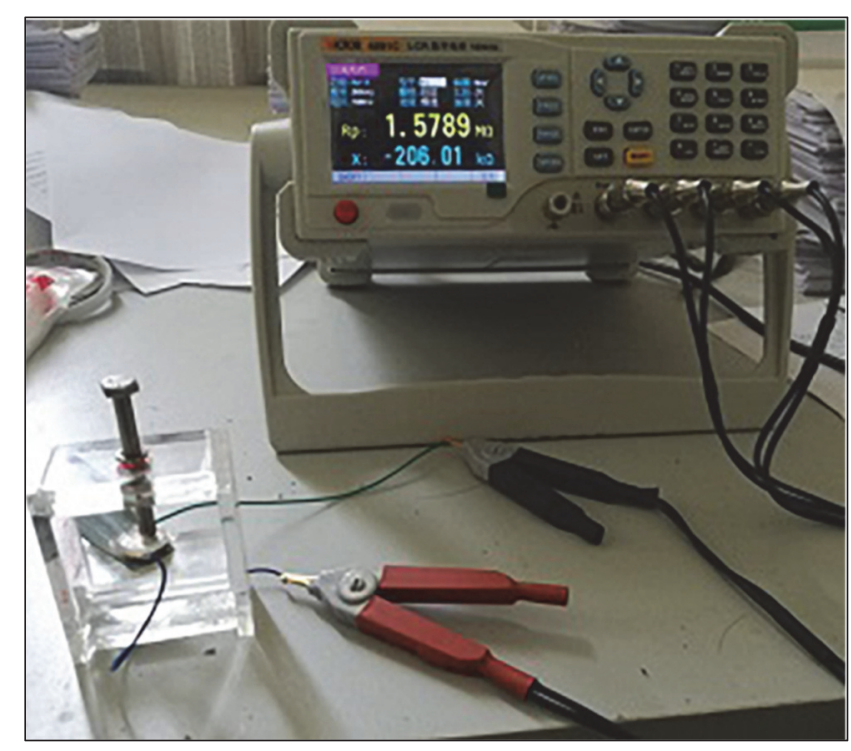

Figure 2. Setup for experimental measurements.

plate up and down. The indication from the pressure sensor was recorded and stabilized for $3 \mathrm{~s}$, and then the LCR meter, which was operated at a calibration voltage of $1 \mathrm{~V}$, was applied. Figure 2 shows the setup used for the experimental measurements.

\section{Determination of Moisture Status}

For the determination of moisture status, the freshly harvested wheat leaves were first weighed with the analytical balance. After measurement of the electrical properties, the leaves were placed in a glass dish, dried in the constant-temperature blast drying oven at $110^{\circ} \mathrm{C}$ for $30 \mathrm{~min}$, and then dried to a constant mass at $80^{\circ} \mathrm{C}$. All measurements were carried out at a constant room temperature of $20^{\circ} \mathrm{C}$ to $24^{\circ} \mathrm{C}$ and relative humidity of $30 \%$ to $35 \%$. The moisture content $(W)$ of the wheat leaves was determined using the following equation:

$$
W(\%)=\frac{W_{f}-W_{d}}{W_{f}} \times 100
$$

where $W_{f}$ and $W_{d}$ are the fresh weight and dry weight, respectively.

The electrical properties of the freshly collected wheat leaves were measured within the frequency range of 0.12 to $100 \mathrm{kHz}$, and the correlation between the moisture content and the electrical properties was assessed. The moisture status of the wheat leaves was mainly within the range of 0.4651 to 0.7830 . According to the different distributions of moisture status, 20 groups of leaves with different moisture status were selected from the experimental samples for further analysis.

\section{Statistical Analysis}

In the statistical analysis, the least-square method and multiple linear regression (MLR) were performed using Excel and SPSS. The least-square method was used to estimate the regression coefficients of equations 2, 3, and 4, and MLR was used to establish the model between the logarithm of 
moisture content and the two electric parameters (capacitance and resistance). The coefficient of determination $\left(\mathrm{R}^{2}\right)$ and root mean square error (RMSE) were used to measure the fitting quality of the model and the accuracy of the moisture content prediction, respectively.

\section{RESULTS AND DISCUSSION RELATIONSHIPS BETWEEN ELECTRICAL Properties AND Moisture Content}

During the measurements, the voltage was $1 \mathrm{~V}$, and the moisture content of the wheat leaves was within the range of $46 \%$ to $79 \%$. First, the relationship between the capacitance and moisture content and between the resistance and moisture content was tested with the least-square method within the range of 0.12 to $100 \mathrm{kHz}$, and the optimal test frequency was found. This method could establish the correlation between moisture content and resistance or between moisture content and capacitance at any frequency, and the correlation was very strong at some frequencies. Furthermore, because there was no correlation between frequency and moisture content, both resistance and capacitance had strong correlations with leaf moisture content at $50 \mathrm{kHz}$, and the correlation coefficients were 0.9246 and 0.9351 , respectively.

When the moisture content was detected using resistance as the single electrical parameter, the RMSE was 3.29\%, and the RMSE was $3.49 \%$ when using capacitance as the single electrical parameter, while the RMSE was lower when using the two-parameter method (for practical applications, the RMSE should be less than $2 \%$ in the moisture range from $40 \%$ to $80 \%$ ). Therefore, we used the two-parameter method consisting of capacitance and resistance to analyze the correlation with moisture content at any frequency. The correlation coefficient was 0.9592 , and the RMSE decreased to $2.68 \%$ at $50 \mathrm{kHz}$. The two-parameter method was sufficient to detect the moisture content of wheat leaves, the error of prediction was closer to $2 \%$, and the prediction was better.

\section{Moisture Content Detection Model}

This study proved that detection of moisture content required two parameters (capacitance and resistance) at the selected frequency of $50 \mathrm{kHz}$. For a single parameter, the moisture content detection model can be expressed as a logarithmic function, as shown in equations 2 and 3 :

$$
\begin{aligned}
& \ln W=\alpha R+\beta \\
& \ln W=a C+b
\end{aligned}
$$

where $W$ is the moisture content (\%), $C$ is the capacitance $(\mathrm{pF}), R$ is the resistance $(\Omega)$, and $\alpha, \beta a$, and $b$, are constants. For the two-parameter method, combining equations 2 and 3 , the moisture content detection model can be expressed as follows:

$$
\ln W=\beta_{0}+\beta_{1} C+\beta_{2} R
$$

where $\beta_{0}, \beta_{1}$, and $\beta_{2}$ are constants.

Figure 3 shows the correlation coefficients between the actual and predicted moisture contents using the least-square

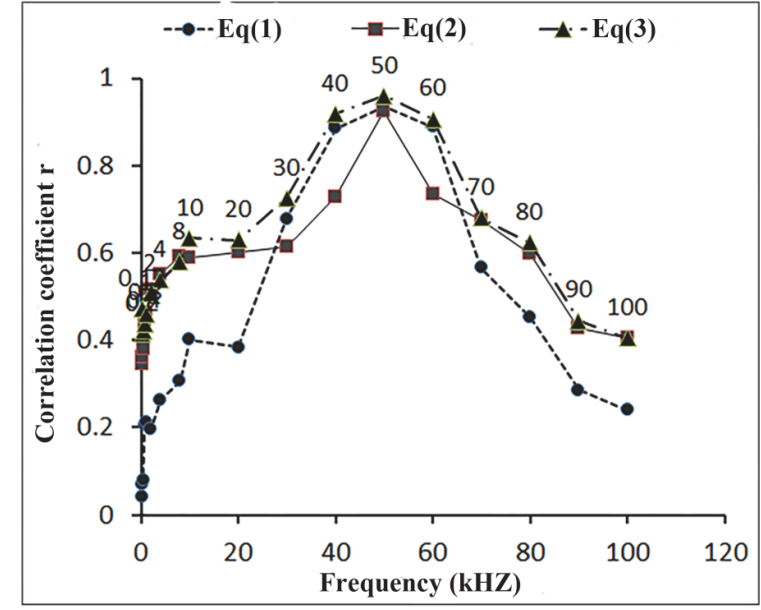

Figure 3. Correlation coefficients between measured and predicted moisture contents by the least-square method obtained based on equations 2,3 , and 4 at different frequencies.

method based on equations 2, 3, and 4 at different frequencies. The correlation was poor in the low-frequency range from 0.12 to $20 \mathrm{kHz}$, which might be because electric current at extremely low frequencies rarely passes through plant tissues (Zhang et al., 1990; Yamada et al., 2003). In the highfrequency range from 80 to $100 \mathrm{kHz}$, the correlation coefficients were all less than 0.68 . The maximum correlation coefficients based on equations 2, 3, and 4 were 0.9246 , 0.9351 , and 0.9592 at $50 \mathrm{kHz}$, respectively. Therefore, 50 $\mathrm{kHz}$ was selected as the optimal frequency for predicting the moisture content of wheat leaves.

The correlation coefficient obtained with equation 4 was higher than the coefficients obtained with equations 2 and 3 . We believed that two parameters would have a stronger effect in detecting moisture content, and the correlation between them was higher, which was helpful for our further research.

The regression analysis between leaf moisture content and the electrical parameters gave the specific expression of equations 2 and 3 under the condition of $50 \mathrm{kHz}$ frequency as follows:

$$
\begin{aligned}
& \hat{y}=5.294-0.7303 R \\
& \hat{y}=3.635+0.0297 C
\end{aligned}
$$

where $\hat{y}$ is the moisture content $(\hat{y}=\ln W)$, expressed as:

$$
W=\exp (\hat{y})
$$

Figure 4 shows the calibration curves between the measured and predicted values of moisture content obtained by equations 5 and 6 . The coefficient of determination $\left(\mathrm{R}^{2}\right)$ between the two equations had a significant difference, and the RMSE values were $3.29 \%$ (for resistance) and 3.49\% (for capacitance). The prediction of moisture content using resistance as the single parameter had a smaller error, a stronger correlation, and higher accuracy. For practical application, the RMSE must be less than $2 \%$. Therefore, to increase the accuracy of prediction, we needed to further improve the model. 

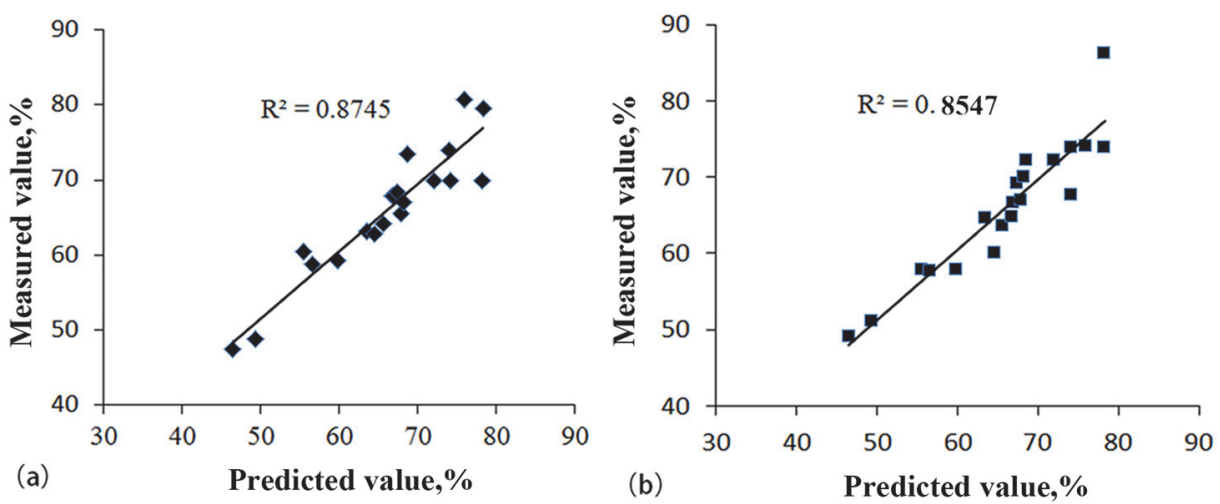

Figure 4. Calibration between measured and predicted values of moisture content obtained by (a) equation 5 and (b) equation 6.

Therefore, an analysis method (MLR) was more suitable for moisture prediction. In predicting the moisture content of tea, MLR obtained a two-parameter prediction model consisting of capacitance and impedance, which had good performance (Mizukami et al., 2006). In this study, the two-parameter method consisting of capacitance and resistance had a strong correlation with the moisture content of wheat leaves at $50 \mathrm{kHz}$ and was superior to the performance of the single-electrical parameter method, with a correlation coefficient as high as 0.9592 . Based on this finding, we chose capacitance and resistance as independent variables and used MLR analysis to obtain equation 8 :

$$
\hat{y}=4.498+0.01503 C-0.3891 R
$$

where $\hat{y}$ is the moisture content $(\hat{y}=\ln W)$, expressed as:

$$
W=\exp (\hat{y})
$$

The results showed a good correlation between the moisture status and the electrical properties of wheat leaves. Figure 5 shows the calibration curve between the measured and predicted values of moisture content obtained with equation 8. From the MLR analysis, the $\mathrm{R}^{2}$ was 0.9154 , and the RMSE was $2.68 \%$. Compared with the linear regression method, the MLR method provided more information, which had a stronger effect on the prediction of moisture content, a higher correlation, and a smaller error, and this was

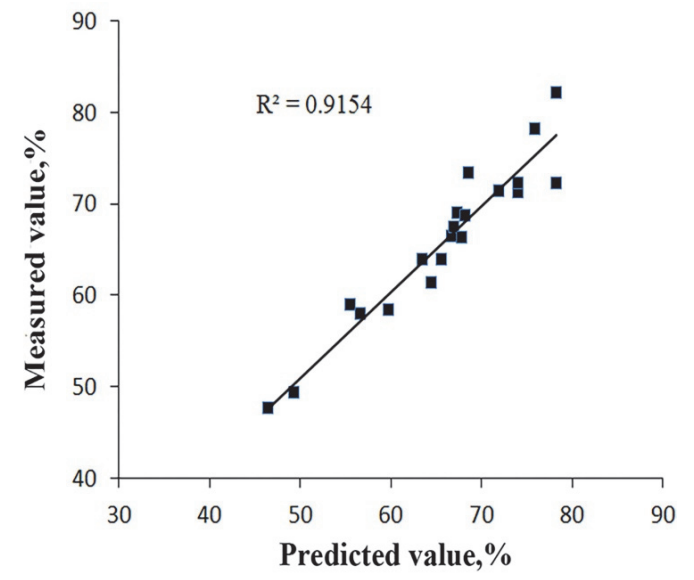

Figure 5. Calibration curve between measured and predicted values of moisture content obtained with equation 8. beneficial for practical application. We also conducted a Student's t-test on the regression coefficients in equation 8 , and the results for the two coefficients were $\mathrm{p}_{1}=0.0105(<0.05)$ and $\mathrm{p}_{2}=0.0075(<0.05)$, indicating statistical significance.

In addition, the standardized partial regression coefficient is the main parameter for comparing the importance of multiple variables, and the standardized partial regression coefficients for capacitance and resistance were 0.479 and 0.505 , respectively. From the absolute values of the two coefficients, resistance had a higher effect on the prediction of moisture content than capacitance in the model. The reason might be that the measurements of capacitance and resistance were affected by different tissues in the leaves, causing different results (Yamada et al., 2003).Therefore, resistance can be considered a more important parameter than capacitance for the prediction of moisture content. Of course, compared with linear regression, the MLR analysis also had disadvantages, including high mathematical complexity, but this drawback did not affect the research. Therefore, we chose equation 8 as the moisture prediction model.

\section{Verification of Prediction Model}

The moisture prediction model was examined using eight leaf samples at two stages (turning-green and jointing), and the results are shown in table 1 . The predicted values of moisture content were higher than the measured values at the turning-green stage, while the predicted values were lower than the measured values at the jointing stage, and the RMSE was also lower. The predicted values of leaf moisture content were closer to the measured values at the turning-green stage. A possible reason was that the data used in the fitting equation mostly came from the turning-green stage, which indicates that a large number of experiments are required to predict plant moisture content by measuring electrical properties.

\begin{tabular}{cccc}
\hline \multicolumn{4}{c}{ Table 1. Verification of leaf moisture content. } \\
\hline Wheat Leaf & $\begin{array}{c}\text { Measured } \\
(\%)\end{array}$ & $\begin{array}{c}\text { Predicted } \\
(\%)\end{array}$ & $\begin{array}{c}\text { RMSE } \\
(\%)\end{array}$ \\
Stage & 51.7 & 52.96 & \\
\multirow{4}{*}{ Turning-green } & 53.94 & 54.4 & 1.25 \\
& 55.37 & 56.83 & \\
\hline \multirow{5}{*}{ Jointing } & 58.6 & 59.38 & \\
& 75.01 & 74.04 & \\
& 76.82 & 75.74 & \\
\hline
\end{tabular}


The method developed in this study for moisture content prediction depends on the electrical properties of leaves (Guo et al., 2007). Nevertheless, pressure also plays a critical role in determining electrical properties. A wheat leaf is a special dielectric material and the measured electrical properties can vary under different pressures (Zheng et al., 2015). Therefore, the pressure between the plates is critically important in the measurement. If the pressure is too low, the leaf will not contact the plate closely, and the air between the plates can reduce the measurement accuracy. In contrast, when the transpiring leaves are cut, the xylem sap retreats in the xylem until it is restricted by the cutting wall. If highpressure is applied to the leaf, the xylem sap will be expressed, which may reduce the volume of water in the leaf (Turner, 1990). When the leaf loses water, the dielectric constant of the leaf decreases, and the capacitance value will decrease (Liu, 2014). Excessive pressure may also damage the leaf tissues and cause measurement errors (Wei et al., 2008). Hence, it was suitable to choose a pressure of $1 \mathrm{~N}$ and observe the leaves with the help of a stereomicroscope (Olympus SZ61). This pressure level allowed the leaf to be in close contact with the plates without damaging the leaf tissues, which lead to the establishment of the prediction model using the leaf electrical properties.

The leaf tissue and temperature can also affect the leaf electrical properties. The equivalent electrical model of plant tissues takes the plasma membrane, cell wall, and extracellular space as a resistor and the cell membrane and cell wall as a capacitor, where moisture exists in the plant body (Mizukami et al., 2006). Therefore, the plant tissue should be taken into account in the measurement of moisture based on electrical properties.

On the one hand, either DC power or AC power can be used for moisture measurement. However, DC power will ionize the water in the leaves, while AC power supply requires determining the optimal frequency (Xuan et al., 2010). The resistance of the plasma membrane and cell wall is very high in the low-frequency range, which is close to DC. Therefore, almost no current passes through the cell gap. However, with an increase of applied frequency, the electric current begins to flow through the extracellular and intracellular spaces because the plasma membrane and cell wall accumulate and release the electric charge in the same way as a capacitor (Zhang et al., 1990; Yamada et al., 2003).

On the other hand, the dielectric constant decreases with the increase of temperature at lower temperatures $\left(-0.5^{\circ} \mathrm{C}\right.$ to $5^{\circ} \mathrm{C}$ ) and increases gradually at higher temperatures (greater than $5^{\circ} \mathrm{C}$ ), reaching the maximum value at about $20^{\circ} \mathrm{C}$. The main reason is that, in dielectric materials with high moisture content, two main factors affect the dielectric loss rate: dipole loss and ion loss. Dipole loss occurs from the rotation of water molecules, which decreases with the increase of temperature at microwave frequencies. Ion loss is due to ion migration, which increases with the increase of temperature. Ion conduction is dominant at low frequencies. However, with the increase of frequency, ion conduction weakens and is replaced by gradually enhanced dipole rotation (Zhao et al., 2018). Based on these considerations, we performed our experimental measurement at room temperature of $20^{\circ} \mathrm{C}$ to $24^{\circ} \mathrm{C}$ to ensure good measurement conditions.

\section{CONCLUSIONS}

In this study, we established a correlation between leaf moisture content and electrical properties at different test frequencies through the collection, analysis, and processing of experimental data for wheat leaves in Southern Xinjiang. The leaf moisture exhibited a remarkable association with the electrical properties at a frequency of $50 \mathrm{kHz}$, and models for moisture prediction of wheat leaves were developed using a single electrical parameter (capacitance or resistance). For both single-parameter models, the RMSE was higher than $2 \%$ within a moisture range from $46 \%$ to $80 \%$. Therefore, the prediction was improved with a two-parameter model (capacitance and resistance). The two-parameter model reduced the prediction error and increased the coefficient of determination.

At the optimal test frequency, a better-fitting equation for leaf moisture content was obtained by using resistance and capacitance, indicating that there was a significant correlation between leaf moisture content and these electrical properties. The resulting equation was more accurate than the single-parameter equations, and the two-parameter equation was chosen as the prediction model. Finally, the model was validated using eight groups of wheat leaf samples at the turning-green stage and jointing stage. Collectively, our findings provide valuable insights into the irrigation efficiency in Southern Xinjiang, promote the development of the regional agricultural economy, and can increase the yield and quality of wheat.

\section{ACKNOWLEDGEMENTS}

The authors declare that there is no conflict of interest that could be perceived as prejudicing the impartiality of the research reported. Authors Yumei Hao and Yuantao Hua performed the research work, interpreted the results, critically analyzed the important data, and drafted the manuscript. Authors Yumei Hao and Jilong Chen contributed to figure drawing and data analysis. Authors $\mathrm{Xu} \mathrm{Li}$ and Xianqiang Gao designed the study, drafted the manuscript, and gave final approval to the version of the manuscript that was submitted for publication. All authors read and approved the final manuscript. The project was supported by the National Natural Science Foundation of China (Grant No. 61765013), the Youth Innovation Fund Project of President Fund of Tarim University (Grant No. TDZKQN201822), and the Innovation and Entrepreneurship Training Program of University Students of Tarim University (Grant No. 2019046).

\section{REFERENCES}

Abayomi, Y. A., \& Wright, D. (1999). Effects of water stress on growth and yield of spring wheat (Triticum aestivum L.) cultivars. Trop. Agric., 76(2), 120-125.

Afzal, A., Mousavi, S. F., \& Khademi, M. (2010). Estimation of leaf moisture content by measuring the capacitance. J. Agric. Sci. Tech.., 12(3), 339-346.

Dean, T. J., Bell, J. P., \& Baty, A. J. B. (1987). Soil moisture measurement by an improved capacitance technique: Part I. Sensor design and performance. J. Hydrol., 93(1), 67-78. https://doi.org/10.1016/0022-1694(87)90194-6 
Dong, B., Shi, L., Shi, C., Qiao, Y., Liu, M., \& Zhang, Z. (2011). Grain yield and water use efficiency of two types of winter wheat cultivars under different water regimes. Agric. Water Mgmt., 99(1), 103-110. https://doi.org/10.1016/j.agwat.2011.07.013

Eller, H., \& Denoth, A. (1996). A capacitive soil moisture sensor. J. Hydrol., 185(1), 137-146. https://doi.org/10.1016/00221694(95)03003-4

Feng, X., Xuan, Q., \& Zhang, W. (2010). Study on non-destructive inspection system for Populus tomentosa Carr. leaf water content [in Chinese]. J. Anhui Agric. Sci., 38(15), 8213-8215.

Gao, L., Hu, C., \& Chen, S. (2005). Leaf water potential characteristics of winter wheat under different irrigation treatments under sprinkler irrigation [in Chinese]. Soil (4), 410414.

Guo, W., Wu, L., \& Wei, Y. (2007). Influence of water loss on physiological and electrical properties of plants [in Chinese]. $J$. Northwest A\&F Univ. Nat. Sci. Ed., 35(4), 186-191.

Guo, X., Zhao, C., \& Kang, S. (2000). Effects of water on winter wheat morphology, physiological characteristics, and yield [in Chinese]. J. Agric. North China, 15, 40-44.

Jin, S., Zhang, S., \& Zheng, R. (1999). Diagnosis of drought from physiological and electrical characteristics of maize [in Chinese]. Acta Agric. Sinica, 15(3), 91-95.

Kandala, C. V. K., Nelson, S. O., \& Lawrence, K. C. (1989). Nondestructive electrical measurement of moisture content in single kernels of corn. J. Agric. Eng. Res., 44, 125-132. https://doi.org/10.1016/S0021-8634(89)80076-9

Khamparia, A., Singh, A., Luhach, A. K., Pandey, B., \& Pandey, D. K. (2020). Classification and identification of primitive kharif crops using supervised deep convolutional networks. Sustain. Comput. Info. Syst., 28, article 100340. https://doi.org/10.1016/j.suscom.2019.07.003

Liu, D. (2014). Design of portable plant leaf moisture content detector. MS thesis. Xi'an, China: Northwest Agricultural and Forestry University.

Liu, F., Shen, S., Li, Y., Li, L., \& Zou, X. (2013). Effects of water stress on photosynthetic characteristics of maize at different growth stages [in Chinese]. Meteorol. Sci., 4, 28-33.

Liu, X. (1994). Differences of diurnal changes in canopy temperature of winter wheat under the water stress condition [in Chinese]. Acta Agric. Univ. Pekinensis, 20(2), 229-232.

Li, T. (2014). Analysis of food security and supply and demand in Southern Xinjiang [in Chinese]. Xinjiang Soc. Sci. Forum, 4, 5156.

Llave, Y., Terada, Y., Fukuoka, M., \& Sakai, N. (2014). Dielectric properties of frozen tuna and analysis of defrosting using a radio-frequency system at low frequencies. J. Food Eng., 139, 19. https://doi.org/10.1016/j.jfoodeng.2014.04.012

Lu, G., Hu, C., \& Chen, S. (2005). Characteristics of leaf water potential of winter wheat with different water treatments under sprinkling irrigation. [in Chinese]. Soils, 37(4), 410-414.

Lu, Y. (2017). Design of crop leaf moisture detector based on multiple electrical parameters [in Chinese]. Hefei, China: Anhui Agricultural University.

Luan, Z., Liu, X., \& Wang, G. (2007). The relationship between leaf capacitance and water content of wheat under water stress [in Chinese]. Acta Botanica Sinica, 27(11), 2323-2327.

Mamnouei, E., Fotouhi, G. R., Esfahani, M., \& Nakhoda, B. (2006). The effects of water deficit on crop yield and the physiological characteristics of barley (Hordeum vulgare L.) varieties. J. Agric. Sci. Tech., 8(3), 211-219.

Mizukami, Y., Sawai, Y., \& Yamaguchi, Y. (2006). Moisture content measurement of tea leaves by electrical impedance and capacitance. Biosyst. Eng., 93(3), 293-299.

https://doi.org/10.1016/j.biosystemseng.2005.12.009
Rascio, A., Rinaldi, M., De Santis, G., Pecchioni, N., Palazzo, G., \& Palazzo, N. (2019). Measurement of leaf lamina moisture with a low-cost electrical humidity sensor: Case study on a wheat water-mutant. BMC Plant Biol., 19(1), article 411. https://doi.org/10.1186/s12870-019-1987-4

Sankar, S., Srinivasan, P., Luhach, A. K., Somula, R., \& Chilamkurti, N. (2020). Energy-aware grid-based data aggregation scheme in routing protocol for agricultural internet of things. Sustain. Comput. Info. Syst., 28, article 100422. https://doi.org/10.1016/j.suscom.2020.100422

Tanentzap, F. M., Stempel, A., \& Ryser, P. (2015). Reliability of leaf relative water content (RWC) measurements after storage: Consequences for in situ measurements. Botany, 93(9), 535-541. https://doi.org/10.1139/cjb-2015-0065

Thompson, R. B., Gallardo, M., Valdez, L. C., \& Fernandez, M. D. (2007). Using plant water status to define threshold values for irrigation management of vegetable crops using soil moisture sensors. Agric. Water Mgmt., 88(1), 147-158. https://doi.org/10.1016/j.agwat.2006.10.007

Turner, N. C. (1990). Plant water relations and irrigation management. Agric. Water Mgmt., 17(1), 59-73. https://doi.org/10.1016/0378-3774(90)90056-5

Wang, B., Zhang, Y., Hao, B., Xu, X., Zhao, Z., Wang, Z., \& Xue, Q. (2016). Grain yield and water use efficiency in extremely late sown winter wheat cultivars under two irrigation regimes in the North China Plain. PLoS One, 11(4), e0153695. https://doi.org/10.1371/journal.pone.0153695

Wang, G., \& Clarke, J. M. (1993). Relationship of excised-leaf water loss and stomatal frequency in wheat. Canadian J. Plant. Sci., 73(1), 93-99. https://doi.org/10.4141/cjps93-012

Wang, J.-Y., Xiong, Y.-C., Li, F.-M., Siddique, K. H., \& Turner, N. C. (2017). Effects of drought stress on morphophysiological traits, biochemical characteristics, yield, and yield components in different ploidy wheat: A meta-analysis. Adv. Agron., 143, 140-170. https://doi.org/10.1016/bs.agron.2017.01.002

Wang, Y., Zhang, L., Gao, M., Tang, J., \& Wang, S. (2013). Temperature- and moisture-dependent dielectric properties of macadamia nut kernels. Food Bioproc. Tech., 6(8), 2165-2176. https://doi.org/10.1007/s11947-012-0898-2

Wei, Y., Li, D., Mu, C., \& Wang, K. (2008). Relationship between the wheat seedling leaf electric property and test frequency and voltage [in Chinese]. Trans. CSAE, 24(5), 160-169.

Xuan, Q., Feng, X., \& Zhang, W. (2010). Study on the relationship between plant leaf capacitance and water content [in Chinese]. Modern Agric. Sci. Tech., 2, 216-221.

Yamada, K., Yamaura, I., Tanaka, K., Yajima, M., Takahashi, N., $\&$ Yamada, K. (2003). Vector locus of an equivalent radius of the root in growing plants. Proc. 20th IEEE Instrumentation Technology Conf. (Vol. 2, pp. 1294-1297). Piscataway, NJ: IEEE. https://doi.org/10.1109/IMTC.2003.1207960

Yang, L., \& Chen, H. (2016). Spatial and temporal variation characteristics of precipitation in Southern Xinjiang [in Chinese]. Renmin Yellow River, 38(3), 18-21.

Zhang, G. (2016). Research on nondestructive detection of moisture content in maize leaves based on dielectric properties. MS thesis. Zhenjiang, China: Jiangsu University.

Zhang, M. I. N., Stout, D. G., \& Willison, J. H. M. (1990). Electrical impedance analysis in plant tissues. J. Exp. Bot., 41(3), 371-380. https://doi.org/10.1093/jxb/41.3.371

Zhang, S., Jin, S., \& Liu, Q. (1999). Current situation and review of crop water deficit diagnosis methods [in Chinese]. J. Jiangsu Univ. Sci. Tech. Nat. Sci. Ed., (2), 1-4.

Zhao, G., Xu, Y., Qiao, C., Ji, C., \& Shi, R. (2018). Analysis of influencing factors of dielectric water content and dielectric constant model [in Chinese]. Eng. Invest., 46(7), 55-61. 
Zheng, L., Wang, Z., Sun, H., Zhang, M., \& Li, M. (2015). Realtime evaluation of corn leaf water content based on the electrical property of leaf. Comput. Electron. Agric., 112, 102-109.

https://doi.org/10.1016/j.compag.2014.11.007

\section{NOMENCLATURE}

$C=$ physiological capacitance of wheat leaves

$R=$ physiological resistance of wheat leaves

RMSE $=$ root mean square error

$W=$ moisture content of wheat leaf

$W_{d}=$ dry weight of wheat leaf

$W_{f}=$ fresh weight of wheat leaf 